\title{
Letter to the Editor Re "Fractional Modeling and SOC Estimation of Lithium-ion Battery"
}

\author{
Rahat Hasan and Jonathan Scott, Senior Member, IEEE
}

A recent paper by Ma et al., claims to estimate the state of charge of Lithium-ion batteries with a fractional-order impedance model including a Warburg and a constant phase element (CPE) with a maximum error of $0.5 \%$ [1]. The proposed equivalent circuit model from [1] is reproduced in figure 1.

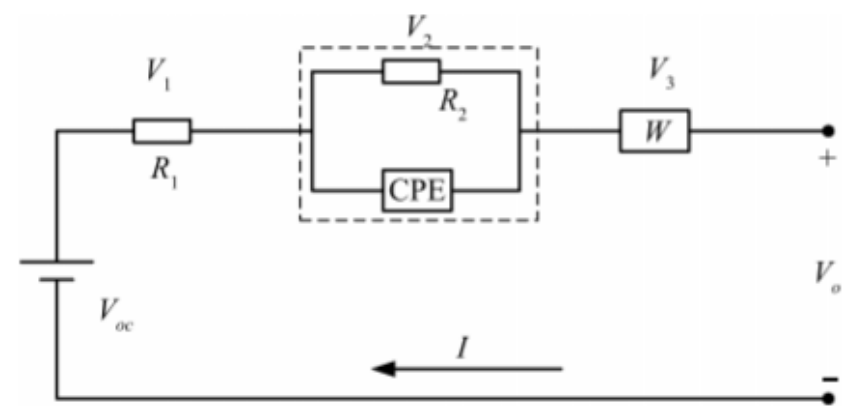

Fig. 1: Fractional equivalent circuit model.

The authors obtain the Laplace-domain impedance of the elements in their model and present this as their equation (12), reproduced here:

$$
H(s)=\frac{V(s)}{I(s)}=\frac{1}{W s^{\alpha}} \times R_{1} \times \frac{R_{2}\left(\frac{1}{C_{2} s^{\beta}}\right)}{R_{2}+\left(\frac{1}{C_{2} s^{\beta}}\right)}
$$

This equation is described as a "transfer function". This is confusing and can mislead readers because it is an impedance (one-port) rather than a transfer (two-port) function. The authors also use $V=V_{o}-V_{o c}$ instead of $V=V_{o c}-V_{o}$, meaning that their equation actually seeks the negative of the impedance, given the direction they have chosen for the conventional current $I(s)$.

More importantly, the equation is simply incorrect. The individual impedance elements are multiplied instead of being summed. The correct equation has addition rather than multiplication signs, so:

$$
\frac{V(s)}{I(s)}=\frac{1}{W s^{\alpha}}+R_{1}+\frac{R_{2}\left(\frac{1}{C_{2} s^{\beta}}\right)}{R_{2}+\left(\frac{1}{C_{2} s^{\beta}}\right)}
$$

In the paper this equation is then carried through to subsequent results. Since the remainder of the paper is based on the initial equation, we must assume that the conclusions are unfounded.

Nevertheless, quite reasonable agreement is obtained when the (erroneous) model is fitted to the data. This might be a result of some numerical co-incidence. Rather, we attribute this outcome to the model having too many degrees of freedom. Almost any reasonable model can be fitted to data within a range given that it has sufficient degrees of freedom. Readers of [1] will note that the model is required only to reproduce data of similar form to that to which the model was originally fitted.

We refer readers to [2] wherein it is shown that a simpler model, with fewer degrees of freedom by virtue of using a single CPE, appears capable of reproducing battery impedance characteristics.

\section{REFERENCES}

[1] Yan Ma, Xiuwen Zhou, Bingsi Li, and Hong Chen, "Fractional Modeling and SOC Estimation of Lithium-ion Battery", IEEE/CAA JOURNAL OF AUTOMATICA SINICA, Vol.3, No. 3, July 2016, pp. 281-287.

[2] Hasan, R., and Scott, J. B., "Fractional behaviour of rechargeable batteries", Proceedings of the 2016 Electronics New Zealand Conference, November 17-18, 2016, Wellington, New Zealand (pp. 111-114). 